\title{
Teste Ergométrico em Crianças e Adolescentes - Maior Tolerância ao Esforço com o Protocolo em Rampa
}

\author{
Treadmill Stress Test in Children and Adolescents: Higher Tolerance on Exertion with Ramp Protocol
}

\author{
Odwaldo Barbosa e Silva, Lurildo C. Ribeiro Saraiva, Dário C. Sobral Filho \\ Hospital das Clínicas - UFPE, Faculdade de Ciências Médicas - UPE - Recife, PE - Brasil
}

\section{Resumo}

Objetivo: Comparar a tolerância ao exercício de crianças e adolescentes submetidos a teste ergométrico (TE) em esteira com os protocolos de Bruce ou em rampa, e descrever a velocidade e a inclinação alcançadas com o protocolo em rampa, para auxiliar na orientação do exercício com esse protocolo.

Métodos: Estudo observacional, tipo série de casos, com controle histórico, de $\mathbf{1 . 0 0 6}$ crianças e adolescentes entre 4 e 17 anos submetidos a TE entre outubro de 1986 e fevereiro de 2003, que concluíram um dos dois protocolos. Foram excluídos os que tiveram o TE interrompido por outras causas que não cansaço físico, os que estavam em uso de medicações que interferiam na freqüência cardíaca (FC) e aqueles com limitações físicas à realização do exercício. Na análise estatística dos dados foi adotado nível de significância para p < 0,05 e intervalo de confiança de 95\%.

Resultados: O tempo de exercício próximo a 10 minutos no protocolo em rampa foi significativamente maior no protocolo de Bruce. A FC máxima alcançada foi superior a $180 \mathrm{bpm}$ nos dois protocolos. A inclinação foi pouco maior nas meninas mais jovens com o protocolo de Bruce, e a velocidade e o consumo máximo de oxigênio ( $\mathrm{VO}_{2}$ max) foram maiores em todas as faixas etárias nos que realizaram o protocolo em rampa.

Conclusão: A velocidade e a inclinação alcançadas com o protocolo em rampa podem ser utilizadas como referência para auxiliar na orientação do exercício no TE com o protocolo em rampa, que mostrou tolerância ao esforço superior à do protocolo de Bruce. (Arq Bras Cardiol 2007;89(6):391-397)

Palavras-chave: Teste de esforço, teste de esteira rolante, criança, adolescente.

Summary

Objective: Compare exercise tolerance by children and adolescents submitted to treadmill stress test (TST) following Bruce Protocol (BP) or Ramp Protocol (RP), as well as describe velocity and inclination reached with ramp protocol to help set protocol exercise standards.

Methods: Observational, case-based study, with history control of 1,006 children and adolescents in the 4 to 17-year-old range who were submitted to TST between October, 1986 and February, 2003, and who concluded one of the two protocols. Those who interrupted their ET for other reasons rather than physical exhaustion, those on medication that interfered in HR and those with physical constraints to exercise were excluded. Statistical analysis of data considered $p<0.05$ as significance level; with confidence interval at $95 \%$.

Results: Exercise time close to 10 minutes in RP was significantly higher than in BP. HR max reached was higher than 180 bpm in both protocols. Inclination showed to be slightly higher in younger girls in Bruce Protocol. Velocity and $\mathrm{VO}_{2}$ max showed to be higher for all age ranges for those in the Ramp Protocol.

Conclusion: Velocity and inclination reached with ramp protocol may be used as reference to help set ramp protocol exercise, which showed superior on exertion tolerance as compared to Bruce protocol. (Arq Bras Cardiol 2007;89(6):354-359)

Key words: Exercise test; child; adolescent.

Full texts in English - http://www.arquivosonline.com.br

\section{Introdução}

O teste ergométrico (TE) é um método diagnóstico com inúmeras indicações ${ }^{1}$ em crianças e adolescentes (idade $\leq 19$ anos $)^{2}$. O protocolo a ser executado deve ser individualizado, de forma que a velocidade e a inclinação da esteira possam ser

Correspondência: Odwaldo Barbosa e Silva •

Rua Dolores Salgado, 219 - Apipucos - 52071-360 - Recife, PE - Brasil E-mail: odwaldo@cardiol.br

Artigo recebido em 28/03/07; revisado recebido em 03/06/07; aceito em 05/06/07. aplicadas de acordo com a capacidade do paciente testado ${ }^{3}$. O TE tem risco muito baixo quando comparado ao realizado em adultos, e complicações não são freqüentes, mesmo quando o teste é realizado em crianças com cardiopatias ${ }^{4,5}$. Conhecimentos específicos de fisiologia do exercício, das diferenças de comportamento da freqüência cardíaca (FC), da pressão arterial (PA) e do eletrocardiograma (ECG), além do uso de esfigmomanômetro de tamanho adequado são prérequisitos à realização do TE nesse grupo de pacientes ${ }^{6}$.

No Brasil, a esteira tem sido utilizada na maioria dos 


\section{Artigo Original}

laboratórios e clínicas que realizam o TE, e o protocolo mais usado é o protocolo de Bruce ${ }^{7}$. O protocolo em rampa caracteriza-se pela duração entre 8 e 12 minutos, com aumentos pequenos e constantes de velocidade e inclinação, individualizados para o sexo e a idade do paciente, tomando como base o consumo máximo de oxigênio ( $\mathrm{VO}_{2}$ max) previsto $^{8-10}$, que pode ser estimado pelas fórmulas do American College of Sports Medicine (ACSM) ${ }^{11,12}$ ou pelo questionário VSAQ (Veterans Specific-Activity Questionnaire) ${ }^{13,14}$.

O objetivo principal foi realizar a comparação das variáveis que avaliam as diferenças da tolerância ao esforço de crianças e adolescentes submetidos aos protocolos de Bruce ou em rampa, para testar a hipótese de que esses dois protocolos são equivalentes. E o objetivo específico foi descrever a velocidade e a inclinação alcançadas com o protocolo em rampa, para auxiliar na orientação do exercício com esse protocolo de acordo com o sexo, a idade e o condicionamento físico do paciente a ser avaliado.

\section{Métodos}

Foi realizado um estudo observacional, tipo série de casos, com controle histórico, de crianças e adolescentes entre 4 e 17 anos, que realizaram o teste ergométrico em esteira ergométrica, em caráter ambulatorial, em clínica na cidade do Recife, segundo um dos dois protocolos: Bruce (controle histórico, TE de outubro de 1986 a fevereiro de 1998) e rampa (março de 1998 a fevereiro de 2003). Foram selecionados apenas os exames interrompidos por fadiga, excluídas outras causas de interrupção ou aqueles em uso de medicações que causam interferências na FC. Foram avaliados apenas os TE realizados em esteira ergométrica por um único examinador, segundo os dois protocolos (Bruce e rampa), tendo sido feita a análise de dados objetivos, que não dependem de interpretação do observador: tempo de exercício, freqüência cardíaca máxima (FC max), velocidade máxima $(\mathrm{km} / \mathrm{h})$, inclinação máxima (em porcentual - \%), e $\mathrm{VO}_{2} \max$ (em mililitros de oxigênio consumido por quilograma de peso corporal por minuto $-\mathrm{ml} \cdot \mathrm{kg}^{-1} \cdot \mathrm{min}^{-1}$ ), calculado pela fórmula para corrida do ACSM ${ }^{11,12}$

Precedendo o esforço, foram realizados anamnese, exame físico, medidas da FC e da PA, e registro do ECG, procedimentos repetidos durante o exercício a cada 3 minutos no protocolo de Bruce e a cada 2 minutos no protocolo em rampa e após o esforço com 1, 2, 4 e 6 minutos, em todos os indivíduos. Os dados foram tabulados no Excel 8.0 Microsoft ${ }^{\circledR}$, e a análise estatística foi realizada utilizando-se o Excel 8.0 e o SigmaStat 3.0. Os resultados estão apresentados em tabelas, categorizadas pelo protocolo do teste, pelo sexo e pelas faixas etárias, distribuídas em quatro classes (4 a 7, 8 a 11, 12 a 14, e 15 a 17 anos). A homogeneidade entre os grupos foi avaliada por idade, peso, altura e FC de repouso por meio do teste t de Student ou do teste de Mann-Whitney (na ausência de distribuição normal ou igualdade das variâncias), e a presença ou ausência de doenças pelo teste do qui-quadrado $\left(\chi^{2}\right)$. O teste de Mann-Whitney foi aplicado para comparação do tempo de esforço, FC max, velocidade, inclinação e $\mathrm{VO}_{2}$ max nos dois protocolos, com significância quando $\mathrm{p}<0,05$ e intervalo de confiança de $95 \%$.
Aspectos éticos - Não foram utilizados protocolos experimentais. O protocolo de Bruce foi introduzido na prática clínica há mais de 50 anos e o protocolo em rampa foi introduzido em 1981 para o cicloergômetro e em 1991 para a esteira ergométrica. Os testes foram solicitados pelos médicos assistentes dos pacientes e realizados por profissional experiente no método, na presença dos pais ou responsáveis, após explicações e orientações sobre o procedimento. Os dados foram coletados dos prontuários dos exames realizados nas crianças e nos adolescentes, e a descrição e a comparação das médias das variáveis categorizadas por protocolo de esforço, sexo e faixa etária garantem o anonimato dos envolvidos no estudo. O projeto foi aprovado pelo Comitê de Ética em Pesquisa Envolvendo Seres Humanos da Instituição.

\section{Resultados}

Foram avaliados 1.006 crianças e adolescentes, 603 (59,9\%) do sexo masculino. Dos 558 (55,5\%) submetidos ao protocolo de Bruce, 58,4\% eram do sexo masculino, e dos $448(45,5 \%)$ que realizaram o protocolo em rampa, 61,8\% eram meninos. Para testar a homogeneidade entre os grupos protocolo de Bruce e protocolo em rampa, foram comparados idade, peso, altura, FC de repouso e presença ou ausência de doença. Não foram observadas diferenças significativas entre idade e altura nos dois sexos. O sexo feminino também não mostrou diferenças quanto ao peso e à FC de repouso. No sexo masculino, apesar de haver diferença significativa do peso e da FC de repouso entre os grupos, quando categorizados por faixa etária, existia distribuição homogênea do peso e a FC de repouso se mostrou ligeiramente mais elevada no protocolo de Bruce apenas na faixa etária entre 15 e 17 anos.

Os grupos não foram homogêneos quanto à presença ou não de doença $(p<0,001)$. No protocolo de Bruce, 495 $(88,7 \%)$ eram sadios e $63(11,3 \%)$ eram doentes (43 com doença cardíaca e 20 asmáticos), comparativamente a 366 $(80,0 \%)$ sadios e $92(20,0 \%)$ doentes (77 cardiopatas e 15 asmáticos) no protocolo em rampa.

Nas meninas, a FC max alcançada foi mais elevada (1 bpm a $4 \mathrm{bpm}$ ) no protocolo de Bruce, com diferença significativa apenas na classe de 8 a 11 anos. O tempo de exercício foi significativamente mais elevado somente entre 12 e 14 anos no protocolo em rampa (tab. 1). Nos meninos, também foram observados valores mais elevados da FC max no protocolo de Bruce (em média, 3 bpm), significativo apenas entre 15 e 17 anos. O tempo de esforço só não se mostrou mais elevado no protocolo de Bruce nas crianças de 4 a 7 anos; para as outras idades, foi significativamente maior (tab. 2).

No sexo feminino, a inclinação máxima no protocolo de Bruce foi maior dos 4 aos 11 anos, menor entre 12 e 14 anos, e sem diferença nas pacientes entre 15 e 17 anos. A velocidade máxima mostrou-se significativamente mais elevada no protocolo em rampa em todas as faixas etárias (tab. 3). No sexo masculino, a inclinação máxima apresentou poucas diferenças, e foi mais elevada no protocolo de Bruce apenas na classe de 12 a 14 anos. A velocidade máxima alcançada foi significativamente maior no protocolo em rampa, em todas as idades (tab. 4). 
Tabela 1 - Freqüência cardíaca máxima (bpm) e tempo de exercício (min:seg) por faixa etária e tipo de protocolo das pacientes do sexo feminino

\begin{tabular}{|c|c|c|c|c|c|c|c|c|c|c|c|c|c|c|}
\hline \multirow{3}{*}{$\begin{array}{l}\text { Protocolo } \\
\text { Faixa etária }\end{array}$} & \multicolumn{7}{|c|}{ FCmax (bpm) } & \multicolumn{7}{|c|}{ Tempo (min:seg) } \\
\hline & \multicolumn{3}{|c|}{ BRUCE } & \multicolumn{3}{|c|}{ RAMPA } & \multirow[t]{2}{*}{ p } & \multicolumn{3}{|c|}{ BRUCE } & \multicolumn{3}{|c|}{ RAMPA } & \multirow[t]{2}{*}{$\mathbf{p}$} \\
\hline & Média & & DP & Média & & DP & & Média & & DP & Média & & DP & \\
\hline $4-7$ & 191,3 & \pm & 10,6 & 188,4 & \pm & 11,8 & 0,613 & 9:41 & \pm & $1: 42$ & $9: 29$ & \pm & $1: 30$ & 0,642 \\
\hline $8-11$ & 194,9 & \pm & 12,0 & 191,0 & \pm & 9,2 & 0,046 & $10: 22$ & \pm & $1: 43$ & 9:50 & \pm & $1: 25$ & 0,062 \\
\hline $12-14$ & 195,1 & \pm & 8,1 & 193,6 & \pm & 7,0 & 0,346 & $9: 47$ & \pm & $1: 48$ & $10: 38$ & \pm & $1: 27$ & 0,014 \\
\hline $15-17$ & 191,3 & \pm & 9,6 & 190,2 & \pm & 7,4 & 0,554 & 10:05 & \pm & $1: 48$ & 10:05 & \pm & $1: 46$ & 0,975 \\
\hline Total & 193,4 & \pm & 10,5 & 191,1 & \pm & 8,8 & 0,018 & $10: 02$ & \pm & $1: 46$ & 10:04 & \pm & $1: 34$ & 0.897 \\
\hline
\end{tabular}

Tabela 2 - Freqüência cardíaca máxima (bpm) e tempo de exercício (min:seg) por faixa etária e tipo de protocolo dos pacientes do sexo masculino

\begin{tabular}{|c|c|c|c|c|c|c|c|c|c|c|c|c|c|c|}
\hline \multirow{3}{*}{$\begin{array}{l}\text { Protocolo } \\
\text { Faixa etária }\end{array}$} & \multicolumn{7}{|c|}{ FCmax (bpm) } & \multicolumn{7}{|c|}{ Tempo (min:seg) } \\
\hline & \multicolumn{3}{|c|}{ BRUCE } & \multicolumn{3}{|c|}{ RAMPA } & \multirow[t]{2}{*}{$\mathbf{p}$} & \multicolumn{3}{|c|}{ BRUCE } & \multicolumn{3}{|c|}{ RAMPA } & \multirow[t]{2}{*}{$\mathbf{p}$} \\
\hline & Média & & DP & Média & & DP & & Média & & DP & Média & & DP & \\
\hline $4-7$ & 187,1 & \pm & 11,4 & 183,4 & \pm & 15,8 & 0,391 & $9: 51$ & \pm & $2: 03$ & $10: 35$ & \pm & $1: 57$ & 0,129 \\
\hline 8-11 & 190,7 & \pm & 10,7 & 187,9 & \pm & 13,8 & 0,144 & $11: 39$ & \pm & $2: 31$ & $10: 27$ & \pm & $1: 43$ & $<0,001$ \\
\hline $12-14$ & 194,7 & \pm & 11,7 & 192,0 & \pm & 12,1 & 0,160 & $12: 44$ & \pm & $3: 03$ & $10: 37$ & \pm & $1: 39$ & $<0,001$ \\
\hline $15-17$ & 194,8 & \pm & 10,0 & 190,2 & \pm & 9,9 & 0,005 & $12: 34$ & \pm & $2: 11$ & 10:39 & \pm & $1: 49$ & $<0,001$ \\
\hline Total & 191,9 & \pm & 11,2 & 189,5 & \pm & 12,8 & 0,023 & $11: 48$ & \pm & $2: 40$ & $10: 34$ & \pm & $1: 44$ & $<0,001$ \\
\hline
\end{tabular}

Tabela 3 - Velocidade $(\mathrm{km} / \mathrm{h})$ e inclinação máxima (\%) por faixa etária e tipo de protocolo das pacientes do sexo feminino

\begin{tabular}{|c|c|c|c|c|c|c|c|c|c|c|c|c|c|c|}
\hline \multirow{3}{*}{$\begin{array}{l}\text { Protocolo } \\
\text { Faixa etária }\end{array}$} & \multicolumn{7}{|c|}{ Velocidade $\max (\mathrm{km} / \mathrm{h})$} & \multicolumn{7}{|c|}{ Inclinação max (\%) } \\
\hline & \multicolumn{3}{|c|}{ BRUCE } & \multicolumn{3}{|c|}{ RAMPA } & \multirow[t]{2}{*}{$\mathbf{p}$} & \multicolumn{3}{|c|}{ BRUCE } & \multicolumn{3}{|c|}{ RAMPA } & \multirow[t]{2}{*}{$\mathbf{p}$} \\
\hline & Média & & DP & Média & & DP & & Média & & DP & Média & & DP & \\
\hline $4-7$ & 6,1 & \pm & 0,9 & 6,6 & \pm & 0,6 & 0,014 & 15,0 & \pm & 1,4 & 14,0 & \pm & 2,0 & 0,011 \\
\hline 8-11 & 6,4 & \pm & 0,8 & 7,2 & \pm & 0,9 & $<0,001$ & 15,6 & \pm & 1,3 & 14,9 & \pm & 2,1 & 0,002 \\
\hline $12-14$ & 6,1 & \pm & 0,9 & 7,8 & \pm & 1,1 & $<0,001$ & 15,1 & \pm & 1,3 & 15,9 & \pm & 2,1 & 0,034 \\
\hline $15-17$ & 6,2 & \pm & 1,0 & 7,7 & \pm & 1,6 & $<0,001$ & 15,3 & \pm & 1,8 & 15,7 & \pm & 2,1 & 0,064 \\
\hline Total & 6,2 & \pm & 0,9 & 7,4 & \pm & 1,2 & $<0,001$ & 15,3 & \pm & 1,5 & 15,2 & \pm & 2,2 & 0,555 \\
\hline
\end{tabular}

Tabela 4 - Velocidade $(\mathrm{km} / \mathrm{h})$ e inclinação máxima (\%) por faixa etária e tipo de protocolo dos pacientes do sexo masculino

\begin{tabular}{|c|c|c|c|c|c|c|c|c|c|c|c|c|c|c|}
\hline \multirow{3}{*}{$\begin{array}{l}\text { Protocolo } \\
\text { Faixa etária }\end{array}$} & \multicolumn{7}{|c|}{ Velocidade max (km/h) } & \multicolumn{7}{|c|}{ Inclinação max (\%) } \\
\hline & \multicolumn{3}{|c|}{ BRUCE } & \multicolumn{3}{|c|}{ RAMPA } & \multirow[t]{2}{*}{$\mathbf{p}$} & \multicolumn{3}{|c|}{ BRUCE } & \multicolumn{3}{|c|}{ RAMPA } & \multirow[t]{2}{*}{$\mathbf{p}$} \\
\hline & Média & & DP & Média & & DP & & Média & & DP & Média & & DP & \\
\hline $4-7$ & 6,2 & \pm & 1,0 & 7,2 & \pm & 1,2 & $<0,001$ & 15,2 & \pm & 1,6 & 15,9 & \pm & 3,0 & 0,283 \\
\hline $8-11$ & 6,9 & \pm & 1,0 & 7,8 & \pm & 1,0 & $<0,001$ & 16,3 & \pm & 1,6 & 16,2 & \pm & 3,1 & 0,128 \\
\hline $12-14$ & 7,3 & \pm & 1,2 & 8,6 & \pm & 1,3 & $<0,001$ & 16,9 & \pm & 1,8 & 16,2 & \pm & 3,1 & 0,002 \\
\hline $15-17$ & 7,2 & \pm & 0,9 & 8,7 & \pm & 1,3 & $<0,001$ & 16,9 & \pm & 1,6 & 17,1 & \pm & 3,4 & 0,703 \\
\hline Total & 7,0 & \pm & 1,1 & 8,2 & \pm & 1,3 & $<0,001$ & 16,4 & \pm & 1,7 & 16,4 & \pm & 3,2 & 0,033 \\
\hline
\end{tabular}




\section{Artigo Original}

Aplicada a fórmula do ACSM (corrida) para o cálculo do $\mathrm{VO}_{2}$ max, apenas as meninas entre 4 e 7 anos de idade não apresentaram diferença significativa entre os grupos Bruce e rampa. Para as demais crianças e adolescentes, foram alcançados valores significativamente mais elevados do $\mathrm{VO}_{2}$ max com o protocolo em rampa, independentemente do sexo ou da idade (tab. 5).

\section{Discussão}

Apesar da baixa prevalência de cardiopatias nas crianças e nos adolescentes, o TE, além de afastar a doença cardíaca e determinar a capacidade funcional, permite ao médico assistente liberar com mais segurança para atividades físicas habituais ou desportivas aqueles com sintomas relacionados ao exercício. O TE normal pode dar aos pais maior tranqüilidade para permitir que seus filhos, doentes ou não, tenham uma vida tão normal quanto possível, sem restrições por vezes desnecessárias de suas atividades físicas.

Pequeno número de publicações descreve o comportamento normal das variáveis hemodinâmicas e metabólicas no TE em crianças e adolescentes, geralmente com os protocolos de Bruce ou em rampa com cicloergômetro, e até mesmo nos trabalhos mais recentes poucos abordam as características específicas dos protocolos do TE. A relevância deste estudo está na apresentação das características do protocolo em rampa com esteira ergométrica em um número significativo de crianças e adolescentes, além de sugerir critérios que podem auxiliar na orientação da velocidade e da inclinação da esteira nesse protocolo, de acordo com o sexo e a idade.

Uma possível limitação deste estudo está na não realização dos dois protocolos em todos os jovens testados, para comparar as reais diferenças de resultados do TE com características diversas. Entretanto, os dois grupos foram homogêneos quanto a idade e altura nos dois sexos, e a FC de repouso e o peso apresentaram pequena diferença apenas nos meninos entre 15 e 17 anos, o que pode garantir maior confiança na comparação dos resultados de dois grupos submetidos aos protocolos diferentes.

As crianças mais jovens costumam ser menos cooperativas na avaliação da capacidade ao exercício máximo. Para compensar as dificuldades em diferenciar a limitação da capacidade em realizar o exercício com a falta de cooperação no teste pediátrico ${ }^{6}$, a experiência do examinador e o encorajamento do jovem, relutante ao exercício máximo, são fatores importantes. O nível de tolerância ao exercício nas crianças pode decorrer mais de fatores motivacionais que de fadiga verdadeira ${ }^{15}$. Essas condições podem justificar o menor tempo de exercício e a menor FC max alcançada pelos mais jovens.

As fórmulas utilizadas para o cálculo da FC max prevista nos adultos não são aplicadas para prever a FC max na população pediátrica. A resposta fisiológica das crianças ao exercício é semelhante à dos adultos, com elevação progressiva proporcional ao aumento da intensidade do esforço, mas difere nos valores máximos alcançados e na menor correlação entre FC e idade. Crianças normais de diferentes faixas etárias atingem FC max acima de 180 bpm, e são comuns valores acima de 200 bpm. Crianças com FC max < 180 bpm ou não foram convenientemente exercitadas ou apresentam déficit cronotrópico ${ }^{16}$.

Nesta série, independentemente do protocolo e do sexo, a FC max alcançada foi superior a $180 \mathrm{bpm}$, o que sugere que todos os adolescentes foram exercitados convenientemente. O protocolo de Bruce demonstrou FC max ligeiramente mais elevada, embora a diferença de $3 \mathrm{bpm}$ a $4 \mathrm{bpm}$ no pico do esforço não tenha significado clínico.

Diversas séries estudadas com o protocolo de Bruce descreveram a FC max: canadenses normais, entre 193 bpm e 206 bpm $^{17}$ e com doença cardíaca, entre 180 bpm e 210 bpm ${ }^{18}$; mexicanos, meninos com 5 anos e meninas com 5 e 6 anos de idade não alcançaram 180 bpm e os demais, entre 184 bpm e 200 bpm ${ }^{19}$; norte-americanos, entre 180 bpm e 200 bpm $^{20}$; napolitanos, $182 \pm 14$ bpm nos meninos e 184 $\pm 13 \mathrm{bpm}$ nas meninas ${ }^{21}$; turcos, $193 \pm 11 \mathrm{bpm}$ nos meninos e $197 \pm 11$ bpm nas meninas ${ }^{22}$.

Poucas publicações abordam crianças normais com o protocolo em rampa na esteira ergométrica. Rowland e Cunningham ${ }^{23}$ estudaram 15 crianças para avaliar o $\mathrm{VO}_{2}$ max e todas ultrapassaram 193 bpm. Török e cols. ${ }^{24}$ observaram 29 meninos normais para comparar com portadores de síndrome plurimetabólica e obesidade isolada, e no grupo normal a FC máxima estava em torno de 185 bpm.

É esperado que na puberdade, com o maior desenvolvimento muscular, os meninos apresentem maior tolerância ao esforço, caracterizada no protocolo de Bruce pelo aumento do tempo de exercício. No protocolo em rampa, o aumento

Tabela $5-\mathrm{VO}_{2} \max \left(\mathrm{ml} \cdot \mathrm{kg}^{-1} \cdot \mathrm{min}^{-1}\right.$ - calculado pela fórmula do ACSM (corrida) por sexo, faixa etária e tipo de protocolo

\begin{tabular}{|c|c|c|c|c|c|c|c|c|c|c|c|c|c|c|}
\hline \multirow{3}{*}{$\begin{array}{l}\mathrm{VO}_{2} \text { max } \\
\text { Protocolo } \\
\text { Faixa etária }\end{array}$} & \multicolumn{7}{|c|}{ Sexo feminino } & \multicolumn{7}{|c|}{ Sexo masculino } \\
\hline & \multicolumn{3}{|c|}{ BRUCE } & \multicolumn{3}{|c|}{ RAMPA } & $\mathbf{p}$ & \multicolumn{3}{|c|}{ BRUCE } & \multicolumn{3}{|c|}{ RAMPA } & \multirow[t]{2}{*}{$\mathbf{p}$} \\
\hline & Média & & DP & Média & & DP & & Média & & DP & Média & & DP & \\
\hline $4-7$ & 37,9 & \pm & 6,4 & 39,4 & \pm & 4,7 & 0,290 & 38,7 & \pm & 7,3 & 45,3 & \pm & 9,2 & 0,018 \\
\hline $8-11$ & 40,3 & \pm & 5,9 & 43,9 & \pm & 6,2 & 0,008 & 43,7 & \pm & 7,5 & 48,6 & \pm & 7,9 & $<0,001$ \\
\hline $12-14$ & 38,0 & \pm & 6,1 & 48,3 & \pm & 7,3 & $<0,001$ & 47,0 & \pm & 9,0 & 53,2 & \pm & 9,0 & $<0,001$ \\
\hline $15-17$ & 38,9 & \pm & 7,9 & 47,8 & \pm & 10,1 & $<0,001$ & 46,4 & \pm & 7,4 & 55,1 & \pm & 9,4 & $<0,001$ \\
\hline Total & 39,0 & \pm & 6,6 & 45,4 & \pm & 8,1 & $<0,001$ & 44,2 & \pm & 8,2 & 51,4 & \pm & 9,3 & $<0,001$ \\
\hline
\end{tabular}


da capacidade física não pôde ser avaliada pelo tempo de exercício, pois o esforço aplicado é maior para se tentar manter o tempo médio de exercício de 10 minutos.

Whipp e cols. ${ }^{25}$ descreveram o tempo ideal como sendo de 4 minutos a 8 minutos para alcançar o esforço máximo no cicloergômetro. Buchfuhrer e cols. ${ }^{26}$ sugeriram duração média de 10 minutos para a tolerância máxima do TE, usando esteira ou cicloergômetro. Testes mais curtos demonstram valores menores do $\mathrm{VO}_{2}$ max, possivelmente por limitação muscular pela maior intensidade do esforço. Da mesma forma, nos testes mais longos, também são encontrados menores valores de $\mathrm{VO}_{2}$ max, decorrentes de aumento da temperatura corporal, de maior desidratação ou de desconforto muscular ou pelos diferentes substratos energéticos envolvidos nos exercícios mais prolongados ${ }^{26}$.

Bozza e Loos ${ }^{16}$ estudaram, com o protocolo de Bruce, 114 brasileiros entre 4 e 18 anos de idade. Nos meninos, o tempo médio de esforço aumentou de 10 minutos com idade $\leq 7$ anos até 13,6 minutos para idade $\geq 15$ anos. Nas meninas, o tempo aumentou de 8,9 minutos nas mais jovens até 12 minutos dos 11 aos 14 anos e caiu acima dos 14 anos. O protocolo de Bruce aplicado em crianças muito jovens ou limitadas pode ter duração muito curta e nas mais treinadas, o tempo pode ultrapassar facilmente os 12 minutos ${ }^{27}$. Cumming e cols. ${ }^{17}$ observaram alta correlação entre o $\mathrm{VO}_{2}$ max e o tempo de exercício, que pode ser usado satisfatoriamente como indicador da tolerância ao esforço no protocolo de Bruce.

No protocolo em rampa, independentemente de sexo, idade ou nível de condicionamento, espera-se duração entre 8 e 12 minutos $^{8-10}$. No protocolo em rampa, como o tempo de exercício esperado é relativamente constante, para se comparar a tolerância ao esforço deste com outros protocolos pode ser utilizado $\mathrm{O}_{2}$ max calculado por uma mesma fórmula. A velocidade e a inclinação máxima alcançadas nesses dois protocolos foi diferente, e apenas para permitir a comparação entre os protocolos de Bruce e em rampa; o $\mathrm{VO}_{2}$ max em todas as crianças e adolescentes foi calculado a partir da inclinação e da velocidade no esforço máximo pelas fórmulas do ACSM para caminhada e corrida, e esta última não mostrou diferença significativa do calculado pela fórmula de Balke (dados não apresentados nos resultados). A unidade usada para corrigir a diferença do peso corporal foi $\mathrm{ml} \cdot \mathrm{kg}^{-1} \cdot \mathrm{min}^{-1}$. Não se pretendeu fazer a classificação funcional desses pacientes pelos valores do $\mathrm{VO}_{2}$ calculado, mas estabelecer uma comparação entre a tolerância ao exercício nesses dois diferentes protocolos de esforço.

De forma semelhante aos outros estudos, $\mathrm{o} \mathrm{VO}_{2}$ max foi maior nos meninos, aumentou com a idade e os valores máximos foram alcançados mais tardiamente no sexo masculino. Embora a maior proporção de indivíduos com doença cardíaca tenha sido observada no protocolo em rampa, em todas as idades o $\mathrm{VO}_{2}$ max foi mais elevado nesse protocolo (protocolo em rampa $=51,4 \pm 9,3$ e protocolo de Bruce $=44,2 \pm 8,2 \mathrm{ml} \cdot \mathrm{kg}^{-1} \cdot \mathrm{min}^{-1}$ ). O encaminhamento mais freqüente de indivíduos nessa faixa etária para a realização do teste ergométrico para avaliação de tratamento clínico ou cirúrgico de cardiopatias congênitas ou valvopatias e avaliação para a prática de atividades físicas poderia justificar o maior número do TE nos últimos anos em crianças e adolescentes com doença cardíaca.

Myers e cols. ${ }^{8}$ encontraram $\mathrm{VO}_{2}$ calculado maior que o medido em todos os protocolos estudados, com menor diferença $(6 \%)$ no protocolo em rampa e maior (16\%) no protocolo de Bruce. Serra ${ }^{9}$ recomenda o protocolo em rampa por permitir melhor identificação do limiar anaeróbio, e maiores níveis de $\mathrm{VO}_{2}$ max. O TE máximo não distingue necessariamente crianças normais daquelas com doença cardíaca de moderada a grave. Muitas crianças com pequenos problemas estruturais do coração, corrigidos ou não, têm $\mathrm{VO}_{2}$ max igual ao de crianças normais. Somente as crianças cianóticas ou com doença valvar grave apresentaram consistente redução da capacidade funcional ${ }^{18}$. Em 7.514 americanos entre 12 e 17 anos de idade, $\mathrm{o} \mathrm{VO}_{2}$ max estimado foi ligeiramente maior na raça negra, aumentou com a idade no sexo masculino e diminuiu no sexo feminino após a puberdade ${ }^{28}$.

Os dados observados nessa população permitiram elaborar a tabela 6, que apresenta a velocidade e a inclinação inicial e aos 10 minutos de exercício, e o $\mathrm{VO}_{2}$ max de acordo com o sexo e a faixa etária, facilitando assim o uso do protocolo em rampa em crianças e adolescentes. Algumas sugestões que se seguem também podem auxiliar na prescrição do exercício no protocolo em rampa: 1 . O tempo previsto de exercício deve ser de 10 minutos - os 2 minutos para mais ou para menos devem acomodar a maioria dos pacientes examinados, dentro do tempo ideal de 8 a 12 minutos de exercício; 2. A velocidade e a inclinação, a serem atingidas aos 10 minutos de exercício, devem ser escolhidas inicialmente, usando como

Tabela 6 - Velocidade $(\mathrm{km} / \mathrm{h})$ e inclinação $(\%)$ sugeridas (inicial e aos 10 minutos) e VO max $\left(\mathrm{ml}_{2} . \mathrm{Kg}^{-1}\right.$. $\left.\mathrm{min}^{-1}\right)$ alcançado por faixa etária e sexo, para orientar a prescrição do exercício no protocolo em rampa

Sexo feminino

Sexo masculino

\begin{tabular}{|c|c|c|c|c|c|c|c|c|c|c|c|c|c|c|}
\hline \multirow{3}{*}{$\begin{array}{l}\text { Faixa etária } \\
4-7\end{array}$} & \multicolumn{2}{|c|}{$\begin{array}{c}\text { Velocidade } \\
\qquad(\mathrm{km} / \mathrm{h})\end{array}$} & \multicolumn{2}{|c|}{ Inclinação (\%) } & \multirow{2}{*}{\multicolumn{2}{|c|}{$\begin{array}{l}\mathrm{VO}_{2} \\
\text { Média }\end{array}$}} & \multirow{3}{*}{$\begin{array}{l}\max \\
\text { DP } \\
4,7\end{array}$} & \multicolumn{2}{|c|}{$\begin{array}{c}\text { Velocidade } \\
(\mathbf{k m} / \mathbf{h})\end{array}$} & \multicolumn{2}{|c|}{ Inclinação (\%) } & \multirow{2}{*}{\multicolumn{2}{|c|}{$\begin{array}{l}\mathrm{VO}_{2} \\
\quad \text { Média }\end{array}$}} & \multirow{3}{*}{$\begin{array}{l}\max \\
\text { DP } \\
9,2\end{array}$} \\
\hline & \multirow{2}{*}{$\begin{array}{c}\text { Inicial } \\
3,0\end{array}$} & \multirow{2}{*}{$\begin{array}{c}10 \text { min } \\
6,5\end{array}$} & \multirow{2}{*}{$\begin{array}{c}\text { Inicial } \\
4,0\end{array}$} & \multirow{2}{*}{$\begin{array}{c}10 \text { min } \\
14,0\end{array}$} & & & & \multirow{2}{*}{$\begin{array}{c}\text { Inicial } \\
3,5\end{array}$} & \multirow{2}{*}{$\begin{array}{c}10 \text { min } \\
7,5\end{array}$} & \multirow{2}{*}{$\begin{array}{c}\text { Inicial } \\
5,0\end{array}$} & \multirow{2}{*}{$\begin{array}{c}10 \text { min } \\
15,0\end{array}$} & & & \\
\hline & & & & & 39,4 & \pm & & & & & & 45,3 & \pm & \\
\hline $8-11$ & 3,5 & 7,0 & 5,0 & 15,0 & 43,9 & \pm & 6,2 & 4,0 & 8,0 & 5,0 & 15,0 & 48,6 & \pm & 7,9 \\
\hline $12-14$ & 4,0 & 8,0 & 5,0 & 15,0 & 48,3 & \pm & 7,3 & 4,0 & 8,5 & 6,0 & 16,0 & 53,2 & \pm & 9,0 \\
\hline $15-17$ & 4,0 & 8,0 & 5,0 & 15,0 & 47,8 & \pm & 10,1 & 4,5 & 9,0 & 6,0 & 16,0 & 55,1 & \pm & 9,4 \\
\hline
\end{tabular}




\section{Artigo Original}

sugestão os dados apresentados na tabela 6, de acordo com o sexo e a idade; 3 . A velocidade inicial deve equivaler a $50 \%$ ou menos da velocidade máxima prevista, para permitir aumento de $0,1 \mathrm{~km} / \mathrm{h}$ a cada 10 ou 15 segundos; 4 . A inclinação inicial, em valores absolutos, deve ser $10 \%$ menor que a inclinação máxima, para permitir aumento da inclinação de $0,5 \%$ a cada 30 segundos; 5 . O período de aquecimento e adaptação ao ergômetro deve ser realizado durante cerca de 2 minutos, com velocidade e inclinação de $50 \%$ dos valores iniciais; 6. A recuperação após o exercício pode ser realizada sem inclinação $(0 \%)$ e velocidade inicial equivalente a $50 \%$ da máxima alcançada, com redução de $10 \%$ a cada 30 segundos. A utilização da forma combinada de pequenos e constantes aumentos da inclinação e da velocidade, durante todo o período de exercício, proporciona maior conforto que o súbito aumento da carga de trabalho, principalmente nos pacientes mais jovens ou do sexo feminino.

\section{Conclusão}

No teste ergométrico em esteira realizado em crianças e adolescentes, a comparação entre os protocolos de Bruce e em rampa demonstrou que os parâmetros descritos $\left(\mathrm{VO}_{2}\right.$ max, velocidade e inclinação) alcançados com o protocolo em rampa podem ser utilizados como referência para auxiliar na prescrição desse protocolo, que demonstrou tolerância ao esforço superior à do protocolo de Bruce.

\section{Potencial Conflito de Interesses}

Declaro não haver conflito de interesses pertinentes.

\section{Fontes de Financiamento}

O presente estudo não teve fontes de financiamento externas.

\section{Vinculação Acadêmica}

Este artigo é parte de tese de mestrado de Medicina Interna de Odwaldo Barbosa e Silva, Dário C. Sobral Filho, Lurildo C. Ribeiro Saraiva pela Universidade Federal de Pernambuco - UFPE e Universidade de Pernambuco - UPE.

\section{Referências}

1. Barbosa e Silva O, Saraiva LCR. Indicações do teste ergométrico em crianças e adolescentes. Rev Bras Med Esporte. 2004;10 (5): 416-9.

2. World Health Organization. Department of Child and Adolescent Health and Development (CAH). [Acesso em 2003 jun 18]. Disponível em: URL: $<$ http://www.who.int/child-adolescent-health>.

3. Barbosa e Silva O, Sobral Filho DC. Uma nova proposta para orientar a velocidade e inclinação no protocolo em rampa na esteira ergométrica. Arq Bras Cardiol. 2003; 81 (1): 42-7.

4. Washington RL, Bricker JT, Alpert BS, Daniels SR, Deckelbaum RJ, Fisher EA, et al. Guidelines for exercise testing in the pediatric age group. From the Committee on Atherosclerosis and Hypertension in Children, Council on Cardiovascular Disease in the Young, the American Heart Association. Circulation. 1994; 90: 2166-79.

5. Freed MD. Exercise testing in children: a survey of techniques and safety. Circulation. 1981;64 (Suppl IV): IV-278.

6. Gibbons RJ, Balady GJ, Beasley JW, Bricker JT, Duvernoy WFC, Froelicher $\mathrm{VF}$, et al. ACC/AHA guidelines for exercise testing: a report of the American College of Cardiology/American Heart Association Task Force on Practice Guidelines (Committee on Exercise Testing). J Am Coll Cardiol. 1997; 30: 260-315.

7. Araújo CGS. Teste de exercício: terminologia e algumas considerações sobre passado, presente e futuro baseado em evidências. Rev Bras Med Esporte. 2000; 6: 77-84

8. Myers J, Buchanan N, Walsh D, Kraemer M, McAuley P, Hamilton-Wessler M, et al. Comparison of the ramp versus standard exercise protocols. J Am Coll Cardiol. 1991; 17: 1334-42.

9. Serra S. Considerações sobre ergoespirometria. Arq Bras Cardiol. 1997; 68: 301-4.

10. Myers J, Froelisher VF. Exercice testing: procedures and implementation. Cardiol Clin. 1993; 11: 199-213.

11. Protocolo em rampa: manual de referência rápida. Brasília, 1998. [Acesso em 2001 fev 28]. Disponível em URL: <http://www.micromed.ind.br/ergopc13/ download/rampa.pdf $>$.

12. Colégio Americano de Medicina do Esporte (ACMS). Manual do ACMS para o teste de esforço e prescrição do exercício: cálculos metabólicos. 5a. ed. Rio de Janeiro: Revinter; 2000. p. 237-50.

13. Myers J, Buchanan N, Smith D, Neutel J, Bowes E, Walsh D, et al. Individualized ramp treadmill: observations on a new protocol. Chest. 1992;101 (Suppl 5): 236-41.

14. Vivacqua R. Considerações sobre o protocolo de rampa aplicado no teste ergométrico. Boletim do Departamento de Ergometria e Reabilitação Cardiovascular da SBC. 1999;18: 16-7.

15. Weindling SN, Wernovsky G, Colan SD, Parker JA, Boutin C, Mone SM, et al. Myocardial perfusion, function and exercise tolerance after the arterial switch operation. J Am Coll Cardiol. 1994; 23: 424-33.

16. Bozza A, Loos L. O teste de esforço em crianças e adolescentes: experiência com brasileiros normais. Rev SOCERJ. 1995; 7: 19-25.

17. Cumming GR, EverattD, Hastman L. Bruce treadmill test in children: normal values in a clinic population. Am J Cardiol. 1978; 41: 69-75.

18. Cumming GR. Maximal exercise capacity of children with heart defects. Am J Cardiol. 1978; 42: 613-9.

19. León JLA, Zajarías A, Vega PF, Medrano G, Buendía A, Attié F. Respuesta de los niños sanos a la prueba de esfuerzo en banda sinfin con el protocolo de Bruce. Arch Inst Cardiol Méx. 1985; 55: 227-33.

20. Paridon SM, Bricker JT. Quantitative QRS changes with exercise in children and adolescents. Med Sci Sports. 1990; 22: 159-64.

21. Maffulli N, Greco R, Greco L, D'Alterio D. Treadmill exercise test in Neapolitan children and adolescents. Acta Paediatr. 1994; 83: 106-12

22. Lenk MK, Alehan D, Celiker A, Alpay F, Sarici Ü. Bruce treadmill test in healthy Turkish children: endurance time, heart rate, blood pressure and electrocardiographic changes. Turkish J Pediatr. 1998; 40: 167-75.

23. Rowland TW, Cunningham LN. Oxygen uptake plateau during maximal treadmill exercise in children. Chest. 1992; 101: 485-9.

24. Török K, Szelényi Z, Pórszász J, Molnár D. Low physical performance in obese adolescent boys with metabolic syndrome. Int J Obes Relat Metab Disord. 2001; 25: 966-70.

25. Wipp BJ, Davis JA, Torres F, Wasserman K. A test to determine parameters of aerobic function during exercise. J Appl Physiol. 1981; 50: 217-21.

26. Buchfuhrer MJ, Hansen JE, Robinson TE, Sue DY, Wasserman K, Whipp BJ. Optimizing the exercise protocol for cardiopulmonary assessment. J Appl Physiol. 1983; 55: 1558-64. 
Barbosa e Silva e cols.

TE com o protocolo em rampa em crianças e adolescentes

\section{Artigo Original}

27. Paridon SM, Alpert BS, Boas SR, Cabrera ME, Caldarera LL, Daniels SR, et al. Clinical stress testing in the pediatric age group: a statement from the American Heart Association Council on Cardiovascular Disease in the Young, Committee on Atherosclerosis, Hypertension, and Obesity in Youth.
Circulation. 2006; 113: 1905-20.

28. Gillum RF. The relationship of treadmill test performance to blood pressure and other cardiovascular risk factors in adolescents. Am Heart J. 1989; 118: $161-71$ 